\title{
Trauma-Associated Leber Hereditary Optic Neuropathy
}

\author{
Supanut Apinyawasisuk ${ }^{\mathrm{a}, \mathrm{b}}$, Jane W. Chan ${ }^{\mathrm{c}}$, and Anthony C. Arnold \\ aUCLA Department of Ophthalmology, Stein Eye Institute, Los Angeles, California, USA; ${ }^{\circ}$ Department of Ophthalmology, King Chulalongkorn \\ Memorial Hospital, Bangkok, Thailand; 'Department of Neurology and Ophthalmology, University of Arizona College of Medicine, Phoenix, \\ Arizona, USA
}

\begin{abstract}
A 19-year-old man developed visual loss in the left eye 1 day following a martial arts kick to the head. Vision worsened over a week, when visual loss was noted in the right eye without further trauma. The fundus was initially normal, but visual field testing showed temporal depression right eye with diffuse depression left eye, and traumatic chiasmopathy was suspected. Magnetic resonance imaging (MRI) of the brain demonstrated an enlarged chiasm with intrinsic signal abnormality, but no enhancement. Treatment with intravenous corticosteroids and hyperbaric oxygen therapy did not result in visual improvement. Ancillary testing for atypical optic neuritis was negative, but testing for LHON was positive for the 11778 mutation. This case raises the question of trauma as a precipitating factor for LHON and illustrates the rare occurrence of intrinsic signal abnormalities of the chiasm in this disorder.
\end{abstract}

\section{ARTICLE HISTORY}

Received 21 April 2016

Revised 25 April 2016

Accepted 25 April 2016

\section{KEYWORDS}

Chiasmal enlargement; LHON; MRI abnormality; trauma
Leber hereditary optic neuropathy (LHON) is a mitochondrial disorder affecting retinal ganglion cells, which results in acute or subacute bilateral central visual loss. Smoking and ethanol abuse are reported factors that may precipitate the onset of visual loss, although the specific mechanism remains unclear. Visual loss in LHON associated with head or eye trauma has rarely been reported, with varying time interval from trauma to visual loss. The appearance of the visual apparatus on magnetic resonance (MR) imaging has generally been described as normal, although isolated cases of optic nerve enhancement and even rarer cases of chiasmal enlargement without enhancement have been described. We document a case of LHON, positive for the 11778 mutation, which presented within 24 hours of head trauma and demonstrated chiasmal enlargement with intrinsic signal abnormality on MR imaging. The patient was initially diagnosed as traumatic chiasmopathy and treated with corticosteroids and hyperbaric oxygen therapy without improvement.

\section{Case report}

A 19-year-old man reported the rapid onset of central visual loss and decreased color sensitivity in the left eye 1 day following a kick to the head in a martial arts class. He was "stunned," but did not suffer loss of consciousness and did not seek medical attention at the time of the injury. He had no viral illness or other systemic or neurologic complaints. Vision in this eye worsened over the next week, at which time central vision in the right eye was noted to be decreased as well, deteriorating over the next week. He did not report pain with eye movement. Family history was negative for eye disease. He was a prior smoker and denied binge ethanol ingestion.

Examination 2 weeks after onset revealed visual acuity of 20/400 right eye, count fingers at 3 feet left eye. The pupils were mildly sluggish bilaterally with no definite relative afferent pupillary defect (RAPD). The examination was otherwise unremarkable, with equivocal optic disc oedema noted in both eyes. Fluorescein angiography was not performed.

CONTACT Anthony C. Arnold, MD $\otimes$ arnolda@jsei.ucla.edu 0 UCLA Department of Ophthalmology, Stein Eye Institute, 100 Stein Plaza, Los Angeles, CA 90095, USA. 

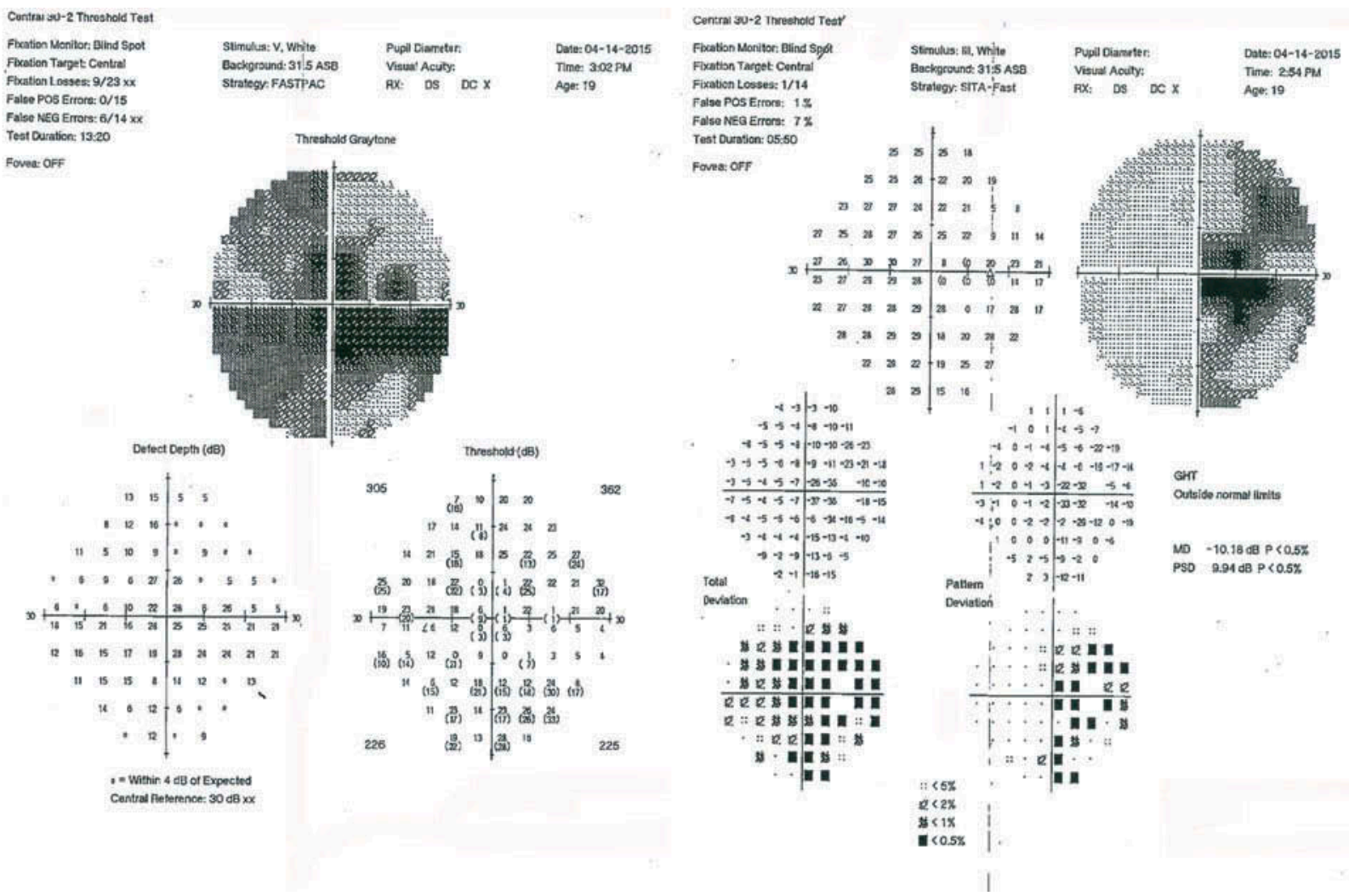

Figure 1. Quantitative perimetry at 2 weeks after initial visual loss, showing cecocentral scotoma with superimposed temporal depression right eye (right), and dense central scotoma with superimposed temporal depression left eye (left).

Quantitative perimetry (Figure 1) demonstrated a cecocentral scotoma right eye with superimposed temporal depression, along with a central scotoma and generalised depression, worse temporally, of the field in the left eye. Ancillary testing included complete blood count (CBC), erythrocyte sedimentation rate (ESR), Venereal Disease Research Laboratory test (VDRL), fluorescent treponemal antibody absorption (FTA-ABS), vitamin $\mathrm{B}_{12}$, and folate levels, all normal. Neuromyelitis optica (NMO) antibody testing was not performed. MR imaging of the brain and orbits showed enlargement of the chiasm, with abnormal intrinsic signal, hyperintense on T2 weighted images, and hypointense on T1-weighted images (Figure 2). The optic nerves appeared normal in size and intensity. Neither the chiasm nor the optic nerves demonstrated abnormal enhancement with gadolinium (Figure 3). An initial diagnosis of
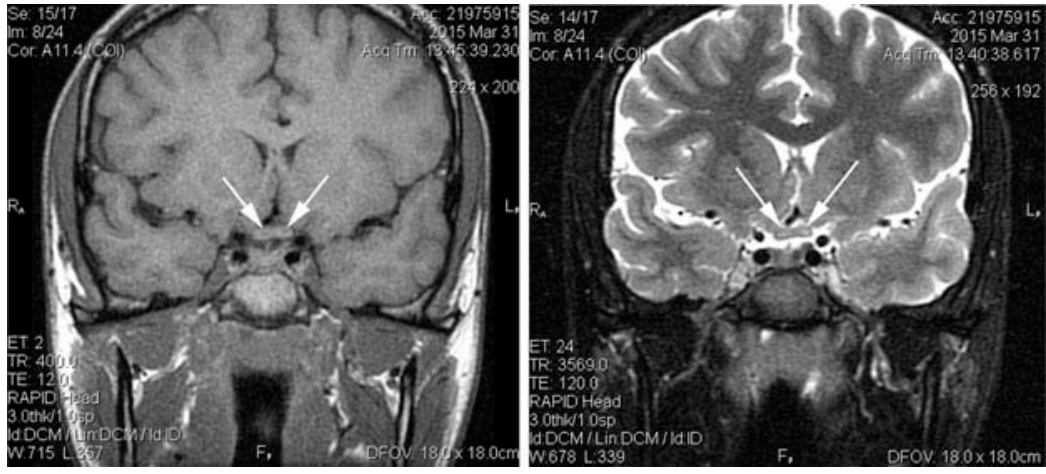

Figure 2. Noncontrast MRI at 2 weeks after initial visual loss. T1-weighted thin coronal section through chiasm (right) demonstrates hypointense central regions bilaterally (arrows). T2-weighted thin coronal section same plane (left) shows hyperintense central regions bilaterally (arrows). 

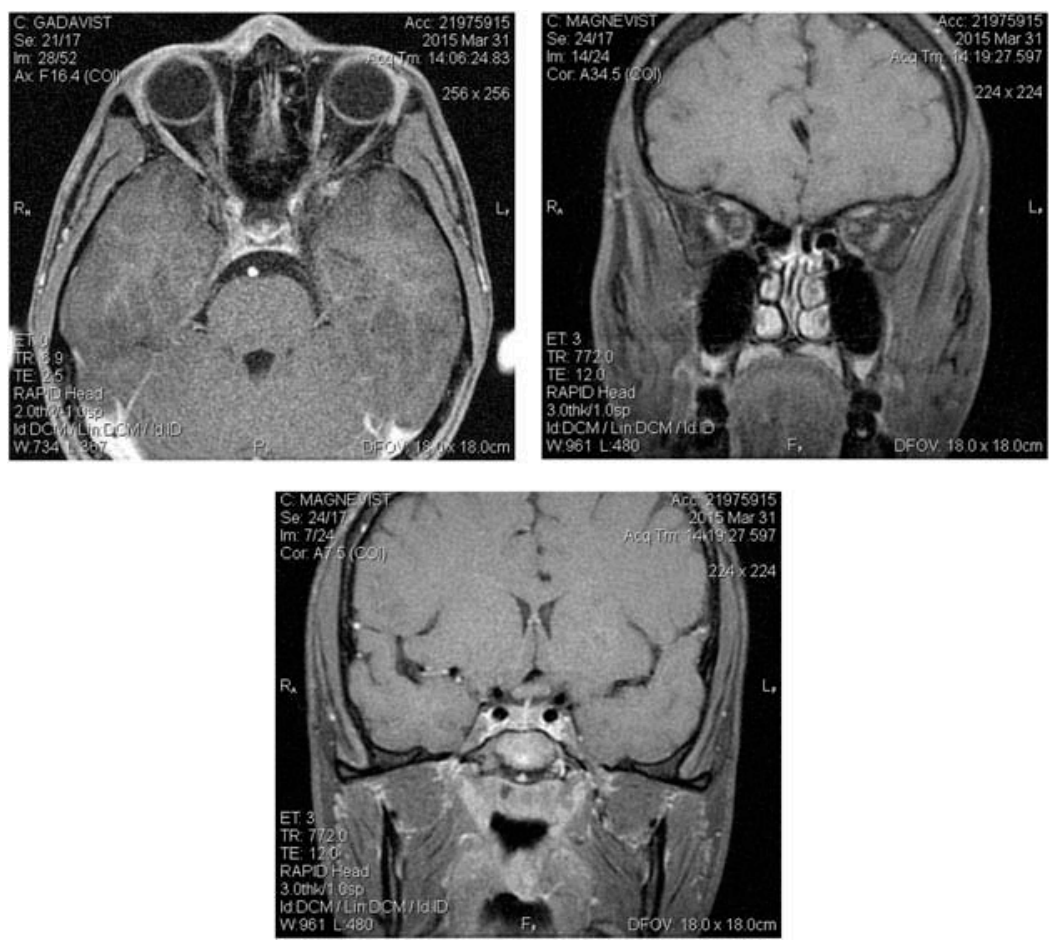

Figure 3. Contrast MRI at 2 weeks after initial visual loss. T1-weighted fat suppression images of intraorbital optic nerves (axial, left, coronal, right) and chiasm (middle) show no enhancement with gadolinium.

traumatic chiasmopathy was made, and the patient received a 2 -month course of oral prednisone $1 \mathrm{mg} /$ $\mathrm{kg} /$ day, followed by a 5-day course of hyperbaric oxygen therapy, but vision did not improve.

Evaluation 4 months after onset showed visual acuity of count fingers at 3 feet right eye, count fingers at 6 feet left eye, no change in perimetry, with $2+$ optic atrophy documented in both eyes. Testing for LHON was positive for the 11778 mutation. MR imaging of the brain performed at this time revealed a decrease in the size of the chiasm, with resolution of the previous intrinsic chiasmal abnormality (Figure 4).

\section{Discussion}

Unaffected LHON carriers may undergo conversion and become symptomatic when they are exposed to certain triggers, including smoking, excessive alcohol consumption, and some
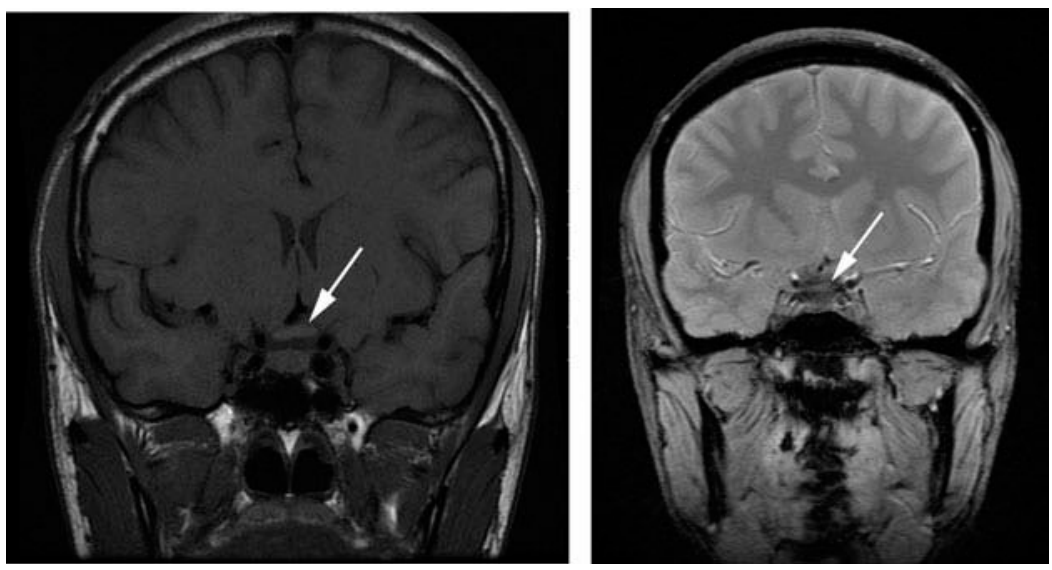

Figure 4. Noncontrast MRI at 4 months after initial visual loss. T1-weighted (left) and T2-weighted (right) thin coronal sections through chiasm show resolution of the previous signal abnormalities and mild decrease in size (arrows). 
antibiotics (macrolides, aminoglycosides, ethambutol, isoniazid, and linezolid). ${ }^{1}$ Head or eye trauma has rarely been reported as a trigger of LHON conversion. Redmill et al. described an 18-year-old man who experienced vision loss 6 days after being punched in the face and was later diagnosed as $\mathrm{LHON}^{2}$ Hayashi and Okamoto described a 34-year-old man who underwent conversion 6 months after head trauma with multiple skull fractures in a traffic accident. ${ }^{3}$ Riordan-Eva et al. reported one posttraumatic LHON patient in their large series; 1 week after being assaulted, with direct trauma to both eyes, he lost vision in one eye, followed 3 weeks thereafter with similar loss in the fellow eye. ${ }^{4}$ Most of these cases were positive for the mitochondrial DNA mutation at position $11778 .^{3,4}$

The specific mechanism for trauma in relation to the conversion of LHON carriers is currently unknown. It has been suggested that a mild traumatic insult may be sufficient to trigger acute decompensation in an optic nerve already compromised by mitochondrial dysfunction. ${ }^{3}$ Levin proposed that superoxide might play an important role in pathogenesis of LHON and other toxic/nutritional optic neuropathies, including ethambutol optic neuropathy. ${ }^{5}$ In LHON, dysfunction of Complex I in the mitochondrial electron transport chain results in excess superoxide production, inducing retinal ganglion cell (RGC) apoptosis. There is evidence of higher superoxide levels in the cerebral extracellular space immediately after experimental traumatic brain injury in an animal model. ${ }^{6}$ We hypothesise that post-traumatic increase in the intracranial superoxide level may trigger retrograde RGC soma apoptosis from the intracranially exposed axonal fibres, inducing conversion in LHON carriers.

MRI of the brain and orbits is typically reported normal in the acute phase of LHON. $^{7,8}$ In our patient, chiasmal enlargement without enhancement, with abnormal intrinsic signal consistent with oedema, was observed at 2 weeks after onset of visual loss. Traumatic chiasmopathy in non-LHON patients typically is characterised by a normal-appearing chiasm on MRI, although rare severe cases may demonstrate structural abnormality. ${ }^{9}$ Moreover, the delayed occurrence of second eye involvement in our case would be inconsistent with this diagnosis. The chiasmal abnormality seen in our patient has also been reported by Phillips et al. in two cases of LHON at 3 weeks and 2 months after the onset of visual loss. ${ }^{10}$ The pathophysiology of chiasmal enlargement in LHON remains unclear. RGC axons in the papillomacular bundle are thought to be more susceptible to superoxide injury due to their greater surface/ volume ratio and greater packing density, with flattening at the site of entry at the temporal optic disc. ${ }^{8}$ Levin ${ }^{5}$ hypothesised that the crossing fibres in the optic chiasm also flatten as they cross under and over each other. In ethambutol optic nerve toxicity, which is also theoretically caused by superoxide's effect, chiasmal involvement resulting in bitemporal hemianopia has been reported in several studies. ${ }^{11-13}$ We propose that axonal fibres in the optic chiasm of LHON carriers might be damaged by the effect of abundant superoxide production similar to the ethambutol optic neuropathy model. The injury may produce swelling in chiasmal axons analogous to that seen in the peripapillary retinal nerve fibre layer (RNFL) of LHON patients. Barboni et al. have proposed that the RNFL thickening observed in the acute phase of LHON is a consequence of both impaired axoplasmic transport and the compensatory increase of mitrochondrial biogenesis. ${ }^{14}$ We hypothesise that chiasmal enlargement might be explained by a similar mechanism. The degree of enlargement and signal abnormality is very mild and easily undetected in routine brain MRI. There are also several reports of contrast enhancement of the optic nerves and chiasm in the acute phase of LHON. Vaphiades et al. reported enhancement of the intraorbital segments of both optic nerves in a LHON patient at 6 weeks after the onset of visual loss. ${ }^{15}$ Lamirel et al. reported prechiasmatic optic nerve enhancement and high signal intensity of the ipsilateral chiasm. ${ }^{16}$ The significance of these findings remains unclear, but they may represent more prominent injury severe enough to result in cell membrane damage and extracellular oedema. 


\section{Declaration of interest}

The authors report no conflicts of interest. The authors alone are responsible for the content and writing of the paper.

\section{Funding}

Supported in part by an unrestricted grant from Research to Prevent Blindness (ACA).

\section{References}

[1] La Morgia C, Carbonelli M, Barboni P, Sadun AA, Carelli V. Medical management of hereditary optic neuropathies. Front Neurol 2014;5(141):1-7.

[2] Redmill B, Mutamba A, Tandon M. Leber's hereditary optic neuropathy following trauma. Eye (Lond) 2001;15 (Pt 4):544-547.

[3] Hayashi S, Okamoto K. [Leber's hereditary optic neuropathy after head trauma: a case report]. Rinsho Shinkeigaku 2011;51:781-783.

[4] Riordan-Eva P, Sanders MD, Govan GG, Sweeney MG, Da Costa J, Harding AE. The clinical features of Leber's hereditary optic neuropathy defined by the presence of a pathogenic mitochondrial DNA mutation. Brain 1995;118(Pt 2):319-337.

[5] Levin LA. Superoxide generation explains common features of optic neuropathies associated with cecocentral scotomas. J Neuroophthalmol 2015;35:152-160.

[6] Kontos HA, Wei EP. Superoxide production in experimental brain injury. J Neurosurg 1986;64:803-807.

[7] Mashima Y, Oshitari K, Imamura Y, Momoshima S, Shiga $\mathrm{H}$, Oguchi Y. Orbital high resolution magnetic resonance imaging with fast spin echo in the acute stage of Leber's hereditary optic neuropathy. J Neurol Neurosurg Psychiatry 1998;64:124-127.

[8] Newman NJ. Leber's hereditary optic neuropathy. New genetic considerations. Arch Neurol 1993;50:540-548.

[9] Segal L, An JA, Gans M. Traumatic disruption of the optic chiasm. J Neuroophthalmol 2009;29:308-310.

[10] Phillips PH, Vaphiades M, Glasier CM, Gray LG, Lee AG. Chiasmal enlargement and optic nerve enhancement on magnetic resonance imaging in leber hereditary optic neuropathy. Arch Ophthalmol 2003;121:577-579.

[11] Kho RC, Al-Obailan M, Arnold AC. Bitemporal visual field defects in ethambutol-induced optic neuropathy. $J$ Neuroophthalmol 2011;31:121-126.

[12] Lim SA. Ethambutol-associated optic neuropathy. Ann Acad Med Singapore 2006;35:274-278.

[13] Osaguona VB, Sharpe JA, Awaji SA, Farb RI, Sundaram AN. Optic chiasm involvement on MRI with ethambutol-induced bitemporal hemianopia. J Neuroophthalmol 2014;34:155-158.

[14] Barboni P, Carbonelli M, Savini G, Ramos Cdo V, Carta A, Berezovsky A, Salomao SR, Carelli V, Sadun AA. Natural history of Leber's hereditary optic neuropathy: longitudinal analysis of the retinal nerve fiber layer by optical coherence tomography. Ophthalmology 2010;117:623-627.

[15] Vaphiades MS, Newman NJ. Optic nerve enhancement on orbital magnetic resonance imaging in Leber's hereditary optic neuropathy. J Neuroophthalmol 1999;19:238-239.

[16] Lamirel C, Cassereau J, Cochereau I, Vignal-Clermont C, Pajot O, Tanguy JY, Zanlonghi X, Reynier P, Amati-Bonneau P, Dubas F, Bonneau D, Verny C. Papilloedema and MRI enhancement of the prechiasmal optic nerve at the acute stage of Leber hereditary optic neuropathy. J Neurol Neurosurg Psychiatry 2010;81:578-580. 